\title{
Understanding the Sociocultural Health Belief Model Influencing Health Behaviors among Saudi Stroke Survivors
}

\author{
Mohammed M. J. Alqahtani \\ King Khalid University, Kingdom of Saudi Arabia \\ Email:mmjbhhal@gmail.com
}

Received 18 September 2015; accepted 29 November 2015; published 2 December 2015

Copyright (C) 2015 by author and Scientific Research Publishing Inc.

This work is licensed under the Creative Commons Attribution International License (CC BY). http://creativecommons.org/licenses/by/4.0/

(c) (7) Open Access

\begin{abstract}
Understanding patients' beliefs of stroke, especially that cultural model which influences the medical management, is important. Professionals normally hold medical knowledge only about stroke, rather than the patients' perceptions, which leads to limitations in cultural competency. Little is known about Saudi stroke survivors' beliefs and behaviors related to their strokes. Methods: A qualitative methodology was used to collect in-depth information from stroke survivors. From March 2010 to October 2014, 45 stroke survivors were recruited from outpatient rehabilitation clinic at King Fahad Medical City (KFMC-RH), during their follow-up treatments. Results: Thematic content analysis yielded four major themes of the causes of stroke: medical, cultural, psychological and environmental. Three major themes were determined regarding stroke interventions: medical, lifestyle and behavioral, as well as cultural interventions. Stroke is most likely to be associated with a wide range of cultural beliefs by stroke survivors, leading them to cultural behaviors and management. All of these cultural interventions were typically used as an adjunct rather than an alternative to medical care. Conclusions: Health professionals need to be aware that cultural beliefs and their management are common problems for stroke survivors undergoing rehabilitation in a hospital. This study could be one step forward to help health professionals become culturally competent when working with stroke survivors in a rehabilitation setting in Saudi Arabia.
\end{abstract}

\section{Keywords}

Stroke, Health Behaviors, Rehabilitation, Cultural Competence, Saudi Arabia

\section{Introduction}

Stroke remains the leading cause of long-term adult disability internationally, and as of this year, the World 
Health Organization (WHO) estimates that more than 50 million healthy persons across the world will be lost as a result of stroke [1]. Stroke is often a life-changing event. Without new information, a patient survivor can be caught in a highly stressful state of unfamiliarity with a new reality [2]. Healthcare professionals frequently teach stroke information based on medical knowledge only, rather than the patients' perceived or actual information needs [3].

Many of the leading causes of disease and death are related to health beliefs and behaviors [4]. Therefore, studies focused on the role of beliefs and behaviors in the promotion of health and the prevention of disease [5]. Several health behavior models have been proposed to understand an individual's health-related behavior. Most of these models assume that patients' health behavior depends upon their beliefs regarding the impact of the illness and its consequences [6]. These models emphasized that a better understanding of the stroke from the patient's perspective allowed for more effective management [7].

Cultural models influence perceptions about the meaning of an illness, the types of treatment that are useful, and the likely outcome of health behaviors related to the prevention and control of a disease [8]. Cultural competence could be seen as a set of attitudes, behaviors and guidelines that came together in a continuum to enable a health care system or health professionals to function effectively in cultural interactions, and to be aware of the dynamics resulting from such cultural differences, and then to adapt services to meet culturally unique needs [9].

Studies on behavior change suggest patients with correct beliefs about their symptoms are more likely to comply with prevention practices, compared to those with incorrect beliefs [10]. Correct beliefs provide a valuable basis to begin behavior modification strategies for stroke recovery [11].

Etiological beliefs about causes of stroke and its management have a significant role in shaping recovery. A poor perception of health post-stroke is linked with lower functional abilities [12] [13]. Understanding poststroke patients' beliefs about their strokes provides a broader picture of how their perceptions impact their recovery.

Stroke terminology includes a large composition of pathophysiological entities that cover thrombosis, embolism, and hemorrhage. Ischemia and hemorrhage are the two major mechanisms causing brain damage in stroke [14]. Stroke rehabilitation ideally includes a returning process involving: (1) assessment, to identify and quantify the patient's needs [15]; (2) definition of an accurate and reasonable goal for improvement; (3) treatment and intervention, and (4) reassessment, to assess progress against agreed goal [16]. The common documented impairment caused by stroke is motor impairment, which limits function in muscle movement or mobility [17]. Other common impairments include those of cognition, speech and language, swallowing, vision and sensation.

Many stroke survivors do not make a full recovery and are left with residual long-term consequences, such as limitation of physical fitness, psychological symptoms, impaired cognition, and other post-stroke symptoms. The available literature emphasizes that rehabilitation providers are not sufficiently aware of stroke survivors' own model of recovery, and of their perceptions of their health behaviors and management post-stroke [18] [19]. Little attention is given to qualitative studies that offer insight to post-stroke patients' views on a model of management and beliefs [20]. Therefore, it is essential to explore patients' beliefs about the causes of stroke, interventions post-stroke, and their model of recovery. Investigation of health knowledge, behavior, and beliefs related to stroke, with special attention given to the culture as an explanatory variable among Saudi, could fill a gap in our existing knowledge and practices. To the best knowledge of the author, there are no qualitative studies from Saudi Arabia or elsewhere in the region regarding belief models influencing health behaviors of stroke survivors.

\section{Method}

A qualitative approach was chosen to gain an in-depth insight into the views and beliefs held by stroke survivors. Qualitative research is the ideal method when the objectives are exploratory, and the hypothesis emerging [21]. The researcher performed a semi-structured interview, with both closed and open questions, to explore patients' beliefs about the etiology and management of their stroke. The semi-structured interviews allowed patients maximum opportunity to express their stories about stroke in terms of their own values and experiences [22]. Interviews were based on a topic guide developed from a literature search about health beliefs and stroke. The interview was piloted with the first participant and was not significantly changed thereafter.

The interviews took place in a quiet room at the rehabilitation hospital, in the outpatient clinics. Interviews were conducted in Arabic and lasted 45 to 60 minutes. 


\subsection{Sample}

The purposive sampling technique, sometimes called judgment sampling, was used, in order to recruit typical patients with definite criteria for exclusion or inclusion. Subjects were recruited from the outpatient rehabilitation clinic, KFMC-RH in Al-Riyadh capital city, Saudi Arabia. To be eligible for the study, patients A) had to have suffered a stroke at least six months prior to be interviewed; B) had to be able to submit consent for interview; C) be normal cognitively, obtaining a minimum score of 27 according to the Montreal Cognitive Assessment (MoCA) [23]. Those with severe dysphasia, confusion and those who were medically unstable due to another medical condition were excluded. Patients were informed the interview was not related to their treatment program. The right to participate and confidentiality were assured. All participating patients signed a written informed consent. Verbal informed consent was documented from those patients who could not sign because of upper-extremity motor impairment. This study was reviewed by the IRB at King Khalid University. Recruitment continued until no new information was found during analysis.

\subsection{Method of Analyzing Data}

The method of analyzing data in the current study was inductive. Data processing following the five-step thematic framework approach [24] which was used to categorize the interview transcripts. This method of analyzing data could be seen in Table 1. Transcripts and categories were examined to ensure the data connected to each category. Data was linked to category headings and agreement was ensured. Quotes were selected to represent and summarize the patient's point of views and to illustrate the variety of beliefs expressed.

\section{Results}

From the interviews, 45 stroke survivors were recruited from the outpatient rehab-psychology clinic, during their follow up (Table 2). They had been discharged from inpatient rehabilitation 6 - 18 months before the interviews.

From the 45 patients, there were 7 patients ( 2 males and 5 females) refused to participate in this study. The reasons given for refusing were having no time and unwillingness. On the other hand, some refused without express reasons. Four patients were interviewed but had not fulfilled the sample's criteria, two because of cognitive limitation ( $\leq 27 \mathrm{MoCA}$ ) and one was dysphasic, and one could not hear well enough to participate.

The final sample was composed of 34 patients, 19 males and 15 females. Age ranged from 37 to 73 . Approximately $80 \%$ of the patients were between 55 and 73 . About two-thirds of the patients completed secondary school or higher, and 11.8\% held postgraduate degrees. A large portion of females held secondary school certificates or below. All patients were married in a stable relationship (i.e. neither divorced, nor separated).

Most of the patients described their health as very good prior to their strokes. In fact, more than half of them were surprised they received a diagnosis of stroke, as they had generally being feeling well before.

"I felt things began to be change in my health. I don't know what happened or came over me, but I was ok." Another patient said, "I have not been ill for a long time, even, you know? I have not been hospitalized before, but overnight I became a customer at clinics.”

Table 1. The five-step thematic framework approach used to categorize the interview transcripts.

\begin{tabular}{|c|c|c|}
\hline & Steps & Analysis and Thematic Framework \\
\hline 1 & Familiarization-immersion & The first step involved familiarization with the raw data in order to list key themes that arose. \\
\hline 2 & $\begin{array}{l}\text { Identifying a } \\
\text { thematic framework }\end{array}$ & $\begin{array}{l}\text { In this step the aim is for drawing out all the main concepts, themes, and issues in relation to the } \\
\text { aims of the study and patient responses. The end product of this step is a detailed index of the } \\
\text { data, which labels the data into manageable chunks for subsequent retrieval and exploration. }\end{array}$ \\
\hline 3 & Indexing & $\begin{array}{l}\text { At this stage all the data was reviewed and the thematic } \\
\text { framework applied; codes were assigned to the relevant themes. }\end{array}$ \\
\hline 4 & Charting & $\begin{array}{l}\text { The data were then rearranged according to the thematic framework. } \\
\text { The charting process involved a considerable amount of abstraction and synthesis. }\end{array}$ \\
\hline 5 & Mapping-interpretation & $\begin{array}{l}\text { This final process involved mapping and interpreting the data, and finding links between themes. } \\
\text { The step of mapping and interpretation is influenced by the original research aims, } \\
\text { as well as by the themes that have emerged from the data themselves. }\end{array}$ \\
\hline
\end{tabular}


Table 2. Demographic profile of the stroke survivors of this sample.

\begin{tabular}{|c|c|c|c|}
\hline \multicolumn{2}{|c|}{ Characteristic } & $n$ & $\%$ \\
\hline \multicolumn{2}{|c|}{ Number of patients approached } & 45 & $(100)$ \\
\hline \multicolumn{2}{|c|}{ Did not agree to participate } & 7 & 15.6 \\
\hline \multirow{3}{*}{ Did not meet the inclusion criteria } & Cognitive limitation & 2 & \\
\hline & Dysphasia & 1 & 8.9 \\
\hline & Other medical & 1 & \\
\hline \multicolumn{2}{|c|}{ Agree to participate and meet the inclusion criteria } & 34 & 100 \\
\hline \multicolumn{2}{|c|}{ Cognitive status (MoCA): Mean ( \pm SD) } & \multicolumn{2}{|c|}{$28.4( \pm 0.8)$} \\
\hline \multirow{4}{*}{ Patient's age category } & $37-44$ & 3 & 8.8 \\
\hline & $45-54$ & 4 & 11.8 \\
\hline & $55-64$ & 14 & 41.2 \\
\hline & $65-73$ & 13 & 38.2 \\
\hline \multirow{2}{*}{ Patient's Gender } & Female & 15 & 44.1 \\
\hline & Male & 19 & 55.9 \\
\hline \multirow{4}{*}{ Patients’s Education } & Illiterate & 5 & 14.7 \\
\hline & Primary or Read \& Write & 16 & 47.1 \\
\hline & Secondary & 11 & 32.4 \\
\hline & Higher & 2 & 5.9 \\
\hline \multirow{3}{*}{ Employment } & Employed & 16 & 47.1 \\
\hline & Unemployed & 9 & 26.5 \\
\hline & Retired & 9 & 26.5 \\
\hline \multirow{3}{*}{ Residence } & Urban & 17 & 50.0 \\
\hline & Semi-rural & 7 & 20.6 \\
\hline & Rural & 10 & 29.4 \\
\hline \multicolumn{2}{|c|}{ Time since stroke in months: Rang; Mean ( \pm SD) } & \multicolumn{2}{|c|}{$6-18 ; 8.4( \pm 2.9)$} \\
\hline \multirow{3}{*}{ Localization } & Right hemisphere & 20 & 58.8 \\
\hline & Left hemisphere & 12 & 35.3 \\
\hline & Other & 2 & 5.9 \\
\hline \multirow{2}{*}{ Pathological subtype } & Ischaemic & 29 & 85.3 \\
\hline & Haemorrhagic & 5 & 14.7 \\
\hline \multirow{5}{*}{ Comorbid conditions } & Hypertension & 18 & 52.9 \\
\hline & Diabetes & 11 & 32.4 \\
\hline & High cholesterol & 13 & 38.2 \\
\hline & Obesity & 8 & 23.5 \\
\hline & Asthma & 5 & 14.7 \\
\hline \multirow{2}{*}{ Mobility } & Walking & 25 & 73.5 \\
\hline & Non-walking & 9 & 26.5 \\
\hline
\end{tabular}




\subsection{Beliefs about Causation}

During the analysis of stroke causes, four themes were classified: medical factors, cultural factors, psychological factors and environmental factors (see Table 3). Few patients had no idea of cause. "No I don't know...really it is a strange disease and I have no clue."

But when the interviews went deeper using prompted questions, patients started expressing their own beliefs, and usually medical explanations emerged first. Hypertension, being overweight, diabetes, and high cholesterol were the most common medical explanations of strokes. "I think it is caused by high blood pressure...you know...most people know that high blood pressure can cause strokes..."

Other patients cited their weight. "I am overweight...I gained too much weight last year...gaining too much weight was my first fault...I lost myself and my health...all these diseases emerge from obesity."

Being overweight was frequently over-cited by patients as a source of their problem.

"My problem is obesity, and the other side of my problem is the side effects of being obese. This obesity brings all the problems into my life..."

All other explanations described external causes, including environmental causes.

“...our fathers and their fathers inhabited here, in this desert for a long, long time without such medical diseases. You know, we have not heard about all of these diseases (before)...these big cities, big workshops, many cares, all of these, you know, have changed our natural life...causing strokes and all of these diseases.”

Interestingly, passive smoking and indoor air pollution were also mentioned as a cause of strokes. "I can say that I was ok until I changed my job... unfortunately I worked with two employees who were smokers, and since then I have had headaches and chest pain...this could cause my stroke..."

Food contamination was reported frequently by patients. “...our food is not healthy...eating meat that contains unacceptably high levels of hormones lead to strokes and cancers.”

The majority of patients stated that they felt their health had deteriorated as a result of stress at work. "I went to my doctor before my health deteriorated...one week before my stroke, and he reassured me that my diabetes and blood pressure were normal and my health in general was good, but it was not! I knew, because I passed through some work and business difficulties...I felt that something burned in my body."

Table 3. This table shows themes and sub-themes about stroke's causes with frequencies and percentages that emerged from qualitative analysis.

\begin{tabular}{|c|c|c|c|}
\hline Themes & Sub-themes & $\mathbf{n}$ & $\%$ \\
\hline \multirow{5}{*}{ Medical factors } & Hypertension & 21 & 61.8 \\
\hline & Overweight & 13 & 38.2 \\
\hline & Diabetes & 9 & 26.5 \\
\hline & High cholesterol & 7 & 20.6 \\
\hline & Other & 1 & 2.9 \\
\hline \multirow{3}{*}{ Spiritual and cultural factors } & By Allah/message from Allah & 26 & 76.5 \\
\hline & Evil eye & 22 & 64.7 \\
\hline & Spirit possession & 3 & 8.8 \\
\hline \multirow{5}{*}{$\begin{array}{l}\text { Psychological and behavior } \\
\text { (lifestyle) factors }\end{array}$} & Food & 27 & 79.4 \\
\hline & Poor physical activities & 20 & 58.8 \\
\hline & Stress, mood and personality & 11 & 32.4 \\
\hline & Smoking & 5 & 14.7 \\
\hline & Other & 2 & 5.9 \\
\hline \multirow[b]{2}{*}{ Environment factors } & Air pollution & 8 & 23.5 \\
\hline & Fond contamination & 6 & 176 \\
\hline
\end{tabular}


Repeatedly, many patients described stress in everyday life as the source of their stroke. "Stroke is one way our body reacts to that stress and changes in our life...I think that my stroke could be linked to pressures I had in my workplace. Stress and pressure mentally, physical and psychologically...ahhhh...all kinds of stress and pressure I had, and went through for more than 22 years in my work."

Some patients reported family and workplace stress together as the source of their strokes. "I have passed through difficult times...I want to change my workplace and escape from my family and I will be ok...because of them, I think that I lost my nervous system, and then I had a stroke."

Family burden was labeled alone as the main source of their strokes. “...My family doesn't understand the cause of my stroke. They put me under pressure all the time... they caused my problem and they don't know this fact..."

Stroke-related behavior and bad health habits were mentioned frequently: "Everyone my age, whether they have diabetes like me or not, should eat a healthful diet that consists of fruits and vegetables. But I have a bad food habit, I don't like the healthy diet...when it comes to eating, I want only our traditional recipes.”

The emphasis was always on food habits and the traditional recipes which contain high sugar, starch and saturated fats. "You know, healthy food habits can help you reduce the risk of having stroke. If I'm serious about protecting myself from another stroke, I have to eat healthy food and lose some weight. I need to shake up our traditional food habits. I need to change those bad eating habits, and start thinking differently about my health."

A sign of clear frustration and self-reproach was observed during interviews where patients admitted their lifestyle and behavior as a reason for their stroke. "Stroke attacks me because of my bad habit of eating everything. I keep eating these fast foods and traditional foods then sleeping...it is a poor way of life."

Overall, the influence of cultural beliefs was notably more reported at the end of each interview. Many more patients believed strokes are attributed to cultural sources. Patients reported several causes behind a stroke. Linking a stroke to Allah's willing always came first. "We are Muslim people and we believe that we have a stroke according to Allah's will...everything that happens comes from Him...so if Allah's will comes it is without cause, as everything that happens comes from Him.”

Interestingly, cultural beliefs could overthrow medical ideas. "Stroke is a disease that is brought by Allah...it caused by our sins... we need to diagnose it not by an MRI, but we need our faith to diagnose our strokes."

Having a stroke is not always a bad things for some patents. "Yes, maybe it was a punishment from Allah. But, when Allah loves someone He may punish him. Allah punishes his slave to remind him of some wrongdoing he may have done."

Both younger and older patients shared this belief. “...Allah tested me to see whether I will be patient or not... and I will please my God.”

In fact, some patients said verbatim that a stroke is good thing. "My stroke is a good sign from Allah...it is not a bad thing... you know sickness comes from Allah in order to wipe out our sins.”

An evil eye represented the most common cultural cause inducing stroke. For example, more than half of the sample believed that strokes are attributed directly to the evil eye. "I think someone has an evil eye on me, I had a mysterious headache then I had a stroke...you know, people's eyes are bad things."

Interestingly, some patients reported when and where they were attacked with the evil eye. "I believe I have an evil eye. I was okay until the summer of 2013, when I attended my friend's wedding ceremony and I sat with a subgroup of the guests around the dinner table. I ate a lot that night and from the next morning I started feeling unwell...I am sure one of them envied me."

Astonishingly, these cultural beliefs were sometimes approved by cultural healers, leading patients to confirm their own cultural model and beliefs about stroke. "I suspected at the beginning about the evil eye, but when I went up to a religious healer he said this condition is not a medical disorder, but rather it is an evil eye."

Post-stroke epilepsy may lead some patients to report some other cultural causes. Three patients thought because of post-stroke epilepsy they suspected the cause of their strokes is spirit possession. "When post-stroke epilepsy came to me, and my wife saw my symptoms during a seizure...she heard me saying unclear words...you know...it was not my voice, but like whispers. She [Wife] said that I was looking and staring at the room ceiling...she said this could be caused by spirits...my stroke could be spirit possession...but you know, doctors don't know these things."

Dramatically, patient beliefs could start with a medical cause and then could shift extremely to a cultural cause, mainly spirit possession. "My stroke started due to medical problems...this is what the doctors said. But unfortunately finding out the cause has not yet solved my problem...really we are confused and frustrated...we 
don't know what happened...stroke did not cause the seizures, am I right?...we don't know what happened...but surely this is a kind of spirit possession."

\subsection{Beliefs about Intervention}

During the analysis of the interventions as perceived by stroke survivors, three themes, as in Table 4, were classified: medical, lifestyle and behavioral, as well as cultural interventions. Same as the scenario above, patients started the conversation usually with medical interventions, and then all of them turned to emphasize the importance of lifestyle and cultural interventions.

Despite differing expectations on their possible cure, almost all patients reported religious practices, such as reading the Quran and praying, as coping strategies for their strokes. Praying was mentioned as the first way to control the physical and psychological features that appear post stroke: "Allah sent this disease to me, and He is able to cure me...when I am ill, it is He who cures me...praying to Allah or donating money to the needy is our way to be cured."

There was tremendous agreement among patients that stroke can be treated via praying and reciting the Quran. "No one can escape from his destiny...this is Allah's will...if we indeed need to be cured, we have to turn our face to seek help from Allah...my prayers and reciting the Quran is my medication."

Some stroke survivors believed skin cauterization was the best cultural intervention for stroke. "I'm telling you I've known people went to a traditional healer for cauterization...he is a famous healer, all stroke patients knew him. My sons took me to him in the first month of my stroke."

Interestingly, this type of cultural intervention is not preferred for female stroke survivors, because skin cauterization may lead to disfigurement. "My husband refused the cauterization, you know...because I am a woman and this thing will sign marks in my body."

Hijama or cupping therapy was reported by some patients. "I asked people who have had experience with hijama...in fact it is a cure for every disease. Hijama, by Allah's permission, can cure all of that pain."

\section{Discussion}

Patients' beliefs were investigated at least six months after their first stroke. This technique gives the sample the advantage of exploring the long-term impact of strokes.

Table 4. This table shows themes and sub-themes about stroke's interventions with frequencies and percentages that emerged from qualitative analysis.

\begin{tabular}{|c|c|c|c|}
\hline Themes & Sub-themes & $n$ & $\%$ \\
\hline \multirow{4}{*}{ Medical (rehabilitation) } & Medications & 32 & 94.1 \\
\hline & Physiotherapy & 28 & 82.4 \\
\hline & Occupational therapy & 11 & 324 \\
\hline & other & 3 & 8.8 \\
\hline \multirow{4}{*}{ Lifestyle and behavior } & Healthy food (Dietary) & 18 & 52.9 \\
\hline & Physical activity & 24 & 70.6 \\
\hline & lose weight & 8 & 23.5 \\
\hline & Avoiding stress & 7 & 20.6 \\
\hline \multirow{5}{*}{ Cultural } & Pray & 28 & 82.4 \\
\hline & Reciting Quran & 25 & 73.5 \\
\hline & Cauterization & 17 & 50.0 \\
\hline & Hijama (Cupping therapy) & 6 & 17.6 \\
\hline & Other & 1 & 2.9 \\
\hline
\end{tabular}


Qualitative methods were selected as the ideal approach for the preliminary exploration of such a complicated issue, and to obtain the deepest understanding of stroke survivors' beliefs. Previous Saudi studies reported the advantage of gathering data from in-depth qualitative interviews [25]. The qualitative design provided a greater understanding of the particular experiences of this population, rather than what could be captured with standardized instruments.

Issues of culture and avoiding contact with men are frequently barriers to collecting information from females in previous Saudi studies (. Including a female psychologist in the current study, in order to collect data from female stroke survivors, avoided such cultural barriers and encouraged female patients to report their beliefs easily.

Saudi Arabia, as a developing country, is undergoing numerous changes. Lifestyles are changing quickly, including changing food habits and decreased physical activities. In Saudi Arabia and neighboring countries, this unhealthy lifestyle increases the prevalence of risk factors for stroke, such as diabetes, hypertension and cardiovascular diseases [26].

It was found that patients tend to be more enthusiastic, particularly when they relate their disease to their lifestyle habits or health-related behavior [6]. Health-related behavior is something they can manage and it could be their own psychological way to cope with their stroke.

Psychological factors were also blamed by most stroke survivors as a cause of their stroke. Patients might have holistic belief systems within which psychological problems are attributed potentially to physical disease [27]. It should be noted here that blaming the psychological element as the source of a stroke, might cause psychological distress itself. Inaccurate beliefs about stroke could be leading a patient to develop anxiety and fear [11]. Psychological, social or environmental needs are given minimal attention during rehabilitation [20] and frequently are not addressed in the early stages of post-stroke.

Stroke survivors acknowledged that the diagnosis of stroke is a wakeup call to absolve oneself from sins. Patients frequently described numerous religious coping strategies, such as praying to Allah or reciting verses from the Quran. Consistent with this result, religiosity has been found to be one of the widespread strategies among stroke patients coping with post-stroke symptoms [28]. As was mentioned above, some patients reported that their stroke could be a good thing. In the Muslim culture, there is a religious belief which sees the person who is in good health for a long time as underprivileged because this indicates that Allah does not want to wipe out his or her sins [29].

The evil eye was also a very common belief. This is regarded as emerging from another person, or rather from the bad soul which inhabits that person. Belief that stroke or other illnesses are caused by the evil eye is widespread across all ethnic and religious groups [30] [31].

A few patients linked their strokes, mainly after they had post-stroke epilepsy, to spirit possession. In spite of increasing health facilities and the rising educational level of patients, the belief in a supernatural existence is widely accepted in the Saudi culture, not only among the illiterate, but also among well-educated people [25] [29]. Believing that one has been possessed or cursed supernaturally might lead stroke survivors to psychological problems [32]. It is also possible that being psychologically distressed makes people more vulnerable to suspect supernatural processes. Alternatively, it is possible that supernatural causes provide a culturally valid way of explaining the problems these patients described [33].

Frequently, the failure of Western medicine to offer a cure or adequate improvements to a stroke condition encouraged people to turn elsewhere for help. A recent survey highlighted that about half of the patients have used traditional interventions, at some point, usually for the treatment of long-term chronic conditions [34]. It has been reported that the common cause for seeking traditional therapies was the lack of a definitive medical cure [29] [34]. However, some use traditional therapies based on their own strong personal beliefs [34].

A Muslim repeatedly seeks help from Allah, prays and recites the Quran in all conditions. The majority of Muslim patients used spiritual healing, such as prayers and reading the Quran for curing all illness [34] [27]. Interestingly, it was reported that religious beliefs and practices are important to many rehabilitation patients after their strokes, because life satisfaction and quality of life can be positively influenced [35].

In the current study, skin cauterization is the third cultural intervention reported by stroke survivors for treating their strokes. Generally, skin cauterization is frequently used in Saudi Arabia to heal chronic medical problems [36]. It is usually performed by firing a bar until it glows red and then cauterizing parts of the body based on underlying causes. In a regional study, stroke survivors [26] reported they preferred to be treated at home with traditional treatments, including cauterizations. 
Hijama was reported by some patients as a traditional process of using a vacuum on different areas of the body in order to gather the blood in that area. It is one of the remedies recommended by the Prophet Muhammad, (Peace Be Upon Him) [37]. Such traditional intervention is commonly used in Saudi Arabia [34].

Traditional interventions in Saudi Arabia are sought by patients for several reasons. Lack of evidence-based scientific data on its effectiveness or safety usually does not prevent Arab patients from flocking to traditional healers [38]. There is a common belief that traditional and herbal medicine is risk-free, and this has led to the development of traditional medicine as the most frequently used [39].

Healthcare in Saudi Arabia has shown remarkable development. However, patients still flock to traditional healers for relief from minor or major health problems, hoping for a permanent cure to their ailments. According to the WHO, more than three-quarters of the world's population depend on traditional medicine [39]. Health professionals have been encouraged to be culturally competent, addressing psychosocial, cultural and spiritual dimensions of the patient [40] [41]; Saha et al., 2008). For example, health professionals in Saudi Arabia should acknowledge that religion affects all aspects of their patients' lives and cannot be separated when delivering medical care and rehabilitation [41].

Despite being a common disorder, stroke is perhaps the neurological condition least well understood by patients and most likely to be associated with a wide range of cultural beliefs.

As seen above, stroke survivors in this study believed that the sources of their strokes were cultural, and their decision for treatment would be according to their beliefs, though this did not mean that they were anti-science. Several patients were looking for management of symptoms rather than a cure [34]. All of these alternative interventions were typically used as an adjunct rather than an alternative to medical care. Traditional interventions, however, could offer hope that Western medicine was unable to provide.

What we need here is cultural competency, understanding the social and cultural influences that affect the quality of medical services and treatment. Cultural competence is a set of behaviors, knowledge, and beliefs that come together in a specific population to enable healthcare to provide effective work in cross-cultural situations [42]. This study recommends that model of Campinha-Bacote [43] which highlights the factor of cultural competence during providing healthcare service. It is a model that views cultural competence as the ongoing procedure to reach the ability of working and providing healthcare service effectively within the cultural context of the patients [44]. This study provides basic contribution to health professionals regarding the importance of culture competence prior to designing any educational or rehabilitation programs [40] for stroke survivors.

\section{Conclusions}

In Arab countries, health professionals have generally been educated very differently from most of their patients [45], or come from different cultural groups. Consequently, they don't have cultural competency or they may not know the kinds of beliefs that their patients have about their strokes.

The current results can help to educate such health professionals about what their patients might believe. Physicians and all rehabilitation professionals who seek to apply the Western model in Arabic culture are at risk of treatment failure [29] [46]. Health professionals need to be aware that cultural beliefs and their management are common problems for stroke survivors undergoing rehabilitation in a hospital. Every effort should be made to fill this gap, and to facilitate better cultural communication and education on stroke rehabilitation units to provide scientific knowledge and proper rehabilitation management.

\section{Limitations}

This study did not screen for psychological improvements or functional recovery, so it is possible that some of the patients' beliefs might have been explained, at least in part, by depression, anxiety or by functional recovery. Future quantitative studies could investigate these factors.

The main limitation was the small number of patients recruited, though for a qualitative study, this is not particularly small. Patients were recruited from KFMC-RH only. But it should be known that KFMC-RH is a tertiary referral hospital which is the main rehabilitation center in Saudi Arabia. It is intended to admit and provide complex rehabilitation care to patients referred from lower level hospitals from all areas of Saudi Arabia.

\section{Acknowledgements}

The author is grateful to the patients who participated in the study, and to the outpatient clinic staff, KFMC-RH, Rehab-Psychology clinic, who helped conduct this study. 


\section{References}

[1] (2005) World Health Organization Preventing Chronic Diseases: A Vital Investment. WHO, Geneva.

[2] Lutz, B.J., Young, M.E., Cox, K.J., Martz, C. and Creasy, K.R. (2011) The Crisis of Stroke: Experiences of Patients and Their Family Caregivers. Topics in Stroke Rehabilitation, 18, 786-797. http://dx.doi.org/10.1310/tsr1806-786

[3] Ang, S.Y., Tin, A.S., Pavitar, G., May, W., Peh, C., Peng, X.J. and Tye, S.N.J. (2013) A Qualitative Study into Stroke Caregivers' Educational Needs-Perspectives of Caregivers and Healthcare Professionals. Proceedings of Singapore Healthcare, 22, 166. http://dx.doi.org/10.1177/201010581302200303

[4] Ezzati, M., Lopez, A.D., Rodgers, A., Vander Hoorn, S. and Murray, C.J. (2002) Selected Major Risk Factors and Global and Regional Burden of Disease. The Lancet, 360, 1347-1360. http://dx.doi.org/10.1016/S0140-6736(02)11403-6

[5] Painter, J.E., Borba, C.P.C., Hynes, M., Mays, D. and Glanz, K. (2008) The Use of Theory in Health Behavior Research from 2000 to 2005: A Systematic Review. Annals of Behavioral Medicine, 35, 358-362. http://dx.doi.org/10.1007/s12160-008-9042-y

[6] Farooqui, M., Hassali, M.A., Shatar, A.K., Shafie, A.A., Seang, T.B. and Farooqui, M.A. (2011) A Qualitative Exploration of Malaysian Cancer Patients’ Perspectives on Cancer and Its Treatment. BMC Public Health, 11, 525. http://dx.doi.org/10.1186/1471-2458-11-525

[7] Rodgers, H., Bond, S. and Curless, R. (2001) Inadequacies in the Provision of Information to Stroke Patients and Their Families. Age and Ageing, 30, 129-133. http://dx.doi.org/10.1093/ageing/30.2.129

[8] Coreil, J., Wilke, J. and Pintado, I. (2004) Cultural Models of Illness and Recovery in Breast Cancer Support Groups. Qualitative Health Research, 14, 905-923. http://dx.doi.org/10.1177/1049732304266656

[9] Ferraro, G.P. (1990) The Cultural Dimension of International Business. Prentice Hall, Englewood Cliffs.

[10] Kreuter, M. and Strecher, V. (1995) Changing Inaccurate Perceptions of Health Risk: Results from a Randomized Trial. Health Psychology, 14, 56-63. http://dx.doi.org/10.1037/0278-6133.14.1.56

[11] Samsa, G.P., Cohen, S.J., Goldstein, L.B., Bonito, A.J., Duncan, P.W., Enarson, C., et al. (1997). Knowledge of Risk among Patients at Increased Risk for Stroke. Stroke, 28, 916-921. http://dx.doi.org/10.1161/01.STR.28.5.916

[12] Hanger, H.C., Fogarty, B., Wilkinson, T.J. and Sainsbury, R. (2000) Stroke Patients Views on Stroke Outcomes: Death versus Disability. Clinical Rehabilitation, 14, 417-424. http://dx.doi.org/10.1191/0269215500cr330oa

[13] Paul, S.L., Sturm, J.W., Dewey, H.M., Donnan, G.A., Macdonell, R.A.L. and Thrift, A.G. (2005) Long-Term Outcome in the North East Melbourne Stroke Incidence Study Predictors of Quality of Life at 5 Years after Stroke. American Heart Association, 36, 2082-2086. http://dx.doi.org/10.1161/01.str.0000183621.32045.31

[14] Saenger, A.K. and Christenson, R.H. (2010) Stroke Biomarkers: Progress and Challenges for Diagnosis, Prognosis, Differentiation, and Treatment. Clinical Chemistry, 56, 21-33. http://dx.doi.org/10.1373/clinchem.2009.133801

[15] Alqahtani, M.M. (2015) Evaluating Patients’ Needs among Rehabilitation Setting. Human, 5, 4-10.

[16] Langhorne, P., Bernhardt, J. and Kwakkel, G. (2011) Stroke Rehabilitation. The Lancet, 377, 1693-1702. http://dx.doi.org/10.1016/S0140-6736(11)60325-5

[17] Langhorne, P., Coupar, F. and Pollock, A. (2009) Motor Recovery after Stroke: A Systematic Review. The Lancet Neurology, 8, 741-754. http://dx.doi.org/10.1016/S1474-4422(09)70150-4

[18] Bendz, M. (2003) The First Year of Rehabilitation after a Stroke-From Two Perspectives. Scandinavian Journal of caring Sciences, 17, 215-222. http://dx.doi.org/10.1046/j.1471-6712.2003.00217.x

[19] Pajalic, Z., Karlsson, S. and Westergren, A. (2006) Functioning and Subjective Health among Stroke Survivors after Discharge from Hospital. Journal of Advanced Nursing, 54, 457-466. http://dx.doi.org/10.1111/j.1365-2648.2006.03844.x

[20] Hartigan, I., O’Connell, E., McCarthy, G. and O’Mahony, D. (2011) First Time Stroke Survivors' Perceptions of Their Status and Their Goals for Recovery. International Journal of Nursing and Midwifery, 3, 22-29.

[21] Britten, N. (1995) Qualitative Research: Qualitative Interviews in Medical Research. BMJ, 311, 251-253. http://dx.doi.org/10.1136/bmj.311.6999.251

[22] Smith, J.A., Harré, R. and Van Langenhove, L., Eds. (1995) Rethinking Methods in Psychology. Sage, London.

[23] Rahman, T.T.A., Gaafary, E. and Mohamed, M. (2009) Montreal Cognitive Assessment Arabic Version: Reliability and Validity Prevalence of Mild Cognitive Impairment among Elderly Attending Geriatric Clubs in Cairo. Geriatrics \& Gerontology International, 9, 54-61. http://dx.doi.org/10.1111/j.1447-0594.2008.00509.X

[24] Pope, C., Ziebland, S. and Mays, N. (2000) Analysing Qualitative Data. BMJ, 320, 114-116. http://dx.doi.org/10.1136/bmj.320.7227.114 
[25] Alqahtani, M.M. (2012) Understanding Autism in Saudi Arabia: A Qualitative Analysis of the Community and Cultural Context. Journal of Pediatric Neurology, 10, 15-22.

[26] Al Shafaee, M.A., Ganguly, S.S. and Al Asmi, A.R. (2006) Perception of Stroke and Knowledge of Potential Risk Factors among Omani Patients at Increased Risk for Stroke. BMC Neurology, 6, 38. http://dx.doi.org/10.1186/1471-2377-6-38

[27] Woloshynowych, M., Valori, R. and Salmon, P. (1998) General Practice Patients’ Beliefs about Their Symptoms. British Journal of General Practice, 48, 885-889.

[28] Donnellan, C., Hevey, D., Hickey, A. and O’Neill, D. (2006) Defining and Quantifying Coping Strategies after Stroke: A Review. Journal of Neurology, Neurosurgery \& Psychiatry, 77, 1208-1218. http://dx.doi.org/10.1136/jnnp.2005.085670

[29] Alqahtani, M.M. and Salmon, P. (2008) Cultural Influences in the Aetiological Beliefs of Saudi Arabian Primary Care Patients about Their Symptoms: The Association of Religious and Psychological Beliefs. Journal of Religion and Health, 47, 302-313. http://dx.doi.org/10.1007/s10943-008-9163-4

[30] Hussain, R. (2002) Lay Perceptions of Genetic Risks Attributable to Inbreeding in Pakistan. American Journal of Human Biology, 14, 264-274. http://dx.doi.org/10.1002/ajhb.10034

[31] Pieroni, A. and Quave, C. (2005) Traditional Pharmacopoeias and Medicines among Albanians and Italians in Southern Italy. Journal of Ethnopharmacology, 101, 258-270. http://dx.doi.org/10.1016/j.jep.2005.04.028

[32] Shahin, S.H. and Daly, E.B. (1999) Knowledge, Attitudes and Beliefs about Psychotropic Medication among Saudi Hospitalized Psychiatric Patients. International Journal of Nursing Studies, 36, 51-55. http://dx.doi.org/10.1016/S0020-7489(98)00057-1

[33] Al-Krenawi, A. and Graham, J.R. (2000) Culturally Sensitive Social Work Practice with Arab Clients in Mental Health Settings. Health \& Social Work, 25, 9-22. http://dx.doi.org/10.1093/hsw/25.1.9

[34] Jan, M.M., Basamh, M.S., Bahassan, O.M. and Jamal-Allail, A.A. (2009) The Use of Complementary and Alternative Therapies in Western Saudi Arabia. Saudi Medical Journal, 30, 682-686.

[35] Giaquinto, S., Spiridigliozzi, C. and Caracciolo, B. (2007) Can Faith Protect from Emotional Distress after Stroke? Stroke, 38, 993-997. http://dx.doi.org/10.1161/01.STR.0000257996.26950.59

[36] Dubovsky, S.L. (1983) Psychiatry in Saudi Arabia. American Journal of Psychiatry, 140, 1455-1459. http://dx.doi.org/10.1176/ajp.140.11.1455

[37] Hasan, I., Ahmad, T. and Ahmad, S. (2014) Management of Hypertension by Wet Cupping Therapy (Al-Hijamah): A Case Study. International Journal of Pharmacology \& Toxicology, 4, 24-27.

[38] Shenoy, R., Bialasiewicz, A., Khandekar, R., Al Barwani, B. and Al Belushi, H. (2009) Traditional Medicine in Oman: Its Role in Ophthalmology. Middle East African Journal of Ophthalmology, 16, 92-96. http://dx.doi.org/10.4103/0974-9233.53869

[39] Azaizeh, H., Saad, B., Cooper, E. and Said, O. (2010) Traditional Arabic and Islamic Medicine: A Re-Emerging Health Aid. Evidence-Based Complementary and Alternative Medicine, 7, 419-424.

[40] Beach, M.C., Price, E.G., Gary, T.L., Robinson, K.A., Gozu, A., Palacio, A., et al. (2005) Cultural Competency: A Systematic Review of Health Care Provider Educational Interventions. Medical Care, 43, 356-373. http://dx.doi.org/10.1097/01.mlr.0000156861.58905.96

[41] Rashidi, A. and Rajaram, S.S. (2001) Culture Care Conflicts among Asian-Islamic Immigrant Women in US Hospitals. Holistic Nursing Practice, 16, 55-64. http://dx.doi.org/10.1097/00004650-200110000-00010

[42] Saha, S., Beach, M.C. and Cooper, L.A. (2008) Patient Centeredness, Cultural Competence and Healthcare Quality. Journal of the National Medical Association, 100, 1275-1285.

[43] Campinha-Bacote, J. (2002) The Process of Cultural Competence in the Delivery of Healthcare Services: A Model of Care. Journal of Transcultural Nursing, 13, 181-184. http://dx.doi.org/10.1177/10459602013003003

[44] Campinha-Bacote, J. (2001) A Model of Practice to Address Cultural Competence in Rehabilitation Nursing. Rehabilitation Nursing, 26, 8-11. http://dx.doi.org/10.1002/j.2048-7940.2001.tb02201.x

[45] Becker, S. (2004) Detection of Somatization and Depression in Primary Care in Saudi Arabia. Social Psychiatry and Psychiatric Epidemiology, 36, 962-966. http://dx.doi.org/10.1007/s00127-004-0835-4

[46] Dwairy, M. and Van Sickle, T. (1996) Western Psychotherapy in Traditional Arabic Societies. Clinical Psychology Review, 16, 231-249. http://dx.doi.org/10.1016/S0272-7358(96)00011-6 